\title{
PROCESOS MISIONALES EN LA GESTIÓN DE CALIDAD DE LA ESCUELA DE POSGRADO DE LA UNIVERSIDAD NACIONAL JOSE FAUSTINO SANCHEZ CARRIÓN, HUACHO-2017
}

\author{
MISSION PROCESSES IN THE QUALITY MANAGEMENT OF THE GRADUATE SCHOOL OF \\ THE NATIONAL UNIVERSITY JOSE FAUSTINO SANCHEZ CARRIÓN, HUACHO-2017
}

\author{
Elvia Mercedes Agurto Tavara ${ }^{1}$, Milagro Rosario Henríquez Suárez ${ }^{2}$, Haydeé Del Rosario, Ramos Pacheco ${ }^{3}$
} Elena Luisa, Laos Fernandez ${ }^{4}$.

\section{RESUMEN}

Objetivo: Identificar los procesos misionales y su influencia en la gestión de calidad de la Escuela de Posgrado de la Universidad Nacional José Faustino Sánchez Carrión, Huacho-2017. Material y Métodos: El diseño de la investigación es no experimental, que nos permitió determinar las características del problema sin alterarlas o cambiarlas. El tipo de investigación utilizado según la finalidad es de tipo básica, descriptiva, correlacional, debido a que se apoyó primeramente en el contexto teórico para luego establecer la correlación frente al problema. Resultado: las actividades de la gestión formativa se relacionan significativamente con la satisfacción de necesidades de la Escuela de Posgrado, las actividades de la gestión administrativa se relaciona significativamente con la satisfacción de necesidades de la Escuela de Posgrado, Las actividades en la gestión de investigación se relacionan significativamente con la satisfacción de necesidades de la Escuela de Posgrado. Conclusión: La identificación de procesos misionales se relaciona significativamente con la gestión de calidad en la Escuela de Posgrado de la Universidad Nacional José Faustino Sánchez Carrión - Huacho-2017.

Palabras clave: Procesos misionale; Gestión de calidad; Escuela de posgrado.

\begin{abstract}
Objective: Identify the mission processes and their influence on the quality management of the school's graduate of the University national José Faustino Sánchez Carrión, Huacho-2017. Material and Methods: Research design is not experimental, which allowed us to determine the characteristics of the problem without altering them or change them. The type of research to used according to the purpose is basic, descriptive, correlational, since I support first of all in the theoretical context to then establish the correlation with the problem. Result: The activities of the formative management are significantly related to the satisfaction of needs of the Graduate School, the activities of the administrative management is significantly related to the satisfaction of needs of the Graduate School, the activities in the research management are relate significantly to the satisfaction of needs of the Graduate School. Conclusion: The identification of mission processes is significantly related to quality management in the Graduate School of the National University José Faustino Sánchez Carrión- Huacho-2017.
\end{abstract}

Key words: Mission processes; Quality management; Graduate school.

\footnotetext{
${ }^{1}$ Doctora en Educación - Universidad Nacional José Faustino Sánchez Carrión. Huacho-Perú. Email: eagurto@unjfsc.edu.pe

${ }^{2}$ Dra. En Administración - Universidad Nacional José Faustino Sánchez Carrión.

${ }^{3}$ M(o). En Salud Pública - Universidad Nacional José Faustino Sánchez Carrión.

${ }^{4}$ Dra. En Ciencias de la Gestión Educativa - Universidad Nacional José Faustino Sánchez Carrión.
} 


\section{INTRODUCCIÓN}

La calidad se refiere a la excelencia relativa de un producto para satisfacer o exceder las necesidades y expectativas razonables de un usuario y en la universidad, como institución del más alto nivel académico, desde su creación en Europa por los siglos XI y XII hasta hoy, ha pasado por una serie de avatares y problemas, hoy, en la era de la globalización y del conocimiento, los problemas de sus fines y de su organización, donde la búsqueda de la calidad va de la mano con las denominadas condiciones básicas de calidad, que sustenta el proceso de licenciamiento institucional de la misma según la regulación vigente.

Existe en la universidad una cultura organizacional anómica que a pesar de existir documentos y normas que sustentan la formalidad, legalidad y el funcionamiento de una institución o grupo humano organizado, hay costumbres, usos, "formas de hacer las cosas", supuestos básicos que no se consignan en ningún documento (Trujillo, 2000) y que, la mayoría de las veces, se constituye en el alma de cualquier sistema social, institucional o empresarial. A esto se le denomina cultura organizacional, de cultura como conocimiento para desarrollar el juicio crítico y como forma de comportamiento habitual o conjunto de costumbres de organización, pensamiento y actuación de los grupos humanos.

Bravo, G. (2005), refiere el rumbo de la universidad en las próximas décadas no tiene una solución lineal, depende de varios factores, algunos de los cuales se encuentran en el interior y otros en el entorno de las universidades. Las trasformaciones posibles del sistema de educación superior y de sus universidades están vinculadas a modelos de organización universitaria en competencia, que a través de su viabilidad y competitividad que permitan su transferencia, combinación y adaptación a las condiciones de un entorno económico y social cambiante, que procuren una educación de calidad, una gestión con calidad y un ejercicio de procesos que favorezcan a los usuarios, como es el caso de los estudiantes de las Maestrías y Doctorados de la Escuela de Posgrado de la Universidad Nacional José Faustino Sánchez Carrión, Huacho, de allí nuestro interés por investigar los procesos misionales en la gestión de calidad de la misma.

La presente investigación tuvo como objetivo identificar los procesos misionales y su relación con la Gestión de calidad de la Escuela de Posgrado de la Universidad Nacional José Faustino Sánchez
Carrión-Huacho-2017

\section{MATERIALES Y MÉTODO}

El diseño de la investigación es no experimental, que nos permitió determinar las características del problema sin alterarlas o cambiarlas. El tipo de investigación a utilizarse según la finalidad es de tipo básica, descriptiva, correlacional, debido a que se apoyó primeramente en el contexto teórico para luego establecer la correlación frente al problema. "Busca especificar las propiedades, las características y los perfiles de personas, grupos, comunidades, procesos, objetos o cualquier otro fenómeno que se someta a un análisis." (Hernández, Fernández y Baptista, 2010).

Asimismo se realizó la investigación haciendo uso del enfoque cuantitativo.

La población está conformada por el personal administrativo que son 10 trabajadores y los estudiantes de maestría que asciende a un total de 660, así mismo 88 estudiantes de doctorado de la escuela de posgrado de la Universidad Nacional José Faustino Sánchez Carrión.

La muestra estuvo conformada por 254 estudiantes de maestría y doctorado a la vez se considerará a los 10 administrativos. La selección de la muestra fue empleando el muestreo aleatorio simple como se muestra a continuación:

Tabla 1. Distribución de la muestra

\begin{tabular}{ccc}
\hline Especialidades & $\begin{array}{c}\text { Número de matriculados } \\
\text { y administrativos }\end{array}$ & $\begin{array}{c}\text { Selección aleatoria } \\
\text { de la muestra }\end{array}$ \\
\hline Maestría & 660 & 224 \\
Doctorado & 88 & 30 \\
Administrativos & 10 & 10 \\
Total & 758 & 264 \\
\hline
\end{tabular}

Técnicas de recolección de datos:

a) Entrevista.- Que nos permitió aplicar la encuesta verificar la información obtenida a través del instrumento de investigación. "Las entrevistas y el entrevistar son elementos esenciales en la vida contemporánea, es comunicación primaria que contribuye a la construcción de la realidad, instrumento eficaz de gran precisión en la medida que se fundamenta en la interrelación humana. Proporciona un excelente instrumento heurístico para combinar los enfoques prácticos, analíticos e interpretativos implícitos en todo proceso de comunicar" (Briones, 2002). 
b) Encuesta.- Para tomar la información que nos llevó a alcanzar los objetivos y la verificación de las hipótesis. "Es un procedimiento que permite explorar cuestiones que hacen a la subjetividad y al mismo tiempo obtener esa información de un número considerable de personas", así por ejemplo: Permite explorar la opinión pública y los valores vigentes de una sociedad, temas de significación científica y de importancia en las sociedades democráticas (Duque, 2013)

c) Bibliográficas.- Que se constituyeron en el soporte para la propuesta de investigación que desarrollamos en el año 2017.

Técnicas para el procesamiento de la información:

a) Estadística descriptiva

Se describieron los datos, los valores de dirección por variables utilizando la distribución de frecuencia y gráficos.

b) Variables

\begin{tabular}{cc}
\hline VARIABLES & DIMENSIONES \\
\hline Independiente Procesos Misionales & Gestión Formativa \\
Gestión Administrativa \\
Gestión de la Investigación \\
Dependiente Gestión de la Calidad & Satisfacción de Necesidades \\
\hline
\end{tabular}

c) Análisis no paramétrico

Para el análisis de las variables con datos nominales $\mathrm{y}$ ordinales aplicándose la prueba ji cuadrado $\mathrm{o} \mathrm{x}^{2}$ para evaluar hipótesis de dos variables categóricas.

d) Paquete estadístico

El análisis estadístico se llevó a cabo mediante el programa SPSS 21 (Statistical Package for the Social Sciences) versión de prueba/e) Instrumento de investigación: Se construyó un cuestionario de satisfacción debidamente estructurado dirigido a la población investigada.

- Construcción de matrices de los procesos misionales correspondiente a la Escuela de Posgrado de la Universidad Nacional José Faustino Sánchez Carrión.

\section{RESULTADOS}

Del instrumento aplicado en el estudio se contrastó las hipótesis para medir la relación de las variables identificación de procesos misionales con la gestión de calidad en la Escuela de Posgrado obteniéndose como resultado lo siguiente:
Hipótesis general

La identificación de procesos misionales se relaciona significativamente con la gestión de calidad en la Escuela de Posgrado de la Universidad Nacional José Faustino Sánchez Carrión- Huacho2017.

Tabla 1. Prueba de Chi-Cuadrado de la Hipótesis General

\begin{tabular}{lc}
\hline \multicolumn{1}{c}{ Estadísticos } & Valor \\
\hline Chi_Cuadrado & 15,75 \\
Sig (bilateral) & 0 \\
Nivel de significancia & 0,05 \\
Coeficiente de contingencia & 0,56 \\
Coeficiente de correlación & 0,78 \\
Número de evaluados & 264 \\
\hline
\end{tabular}

En la tabla 1 se observa la correlación entre la variable procesos misionales y gestión de calidad el cual fue 0,78 lo que indicó una alta correlación positiva y significativa, así mismo al comparar la sig.(bilateral) $\mathrm{p}$ valor $=0$ con el valor del nivel de significancia alfa $=0,05$ se observó que este último es mayor, llegando a la conclusión de rechazo de la hipótesis nula, se concluyó que la identificación de procesos misionales se relaciona significativamente con la gestión de calidad en la Escuela de Posgrado de la Universidad Nacional José Faustino Sánchez Carrión, Huacho - 2017.

Tabla 2. Prueba Chi cuadrado de la Gestión Formativa

\begin{tabular}{lc}
\hline \multicolumn{1}{c}{ Estadísticos } & Valor \\
\hline Chi_Cuadrado & 14,55 \\
Sig (bilateral) & 0 \\
Nivel de significancia & 0,05 \\
Coeficiente de contingencia & 0,48 \\
Coeficiente de correlación & 0,75 \\
Número de evaluados & 264 \\
\hline
\end{tabular}

En la tabla 2 se observa la correlación entre la dimensión gestión formativa y satisfacción de necesidades el cual fue 0,75 lo que indicó una alta correlación positiva y significativa, así mismo al comparar la sig.(bilateral) $\mathrm{p}$ valor $=0$ con el valor del nivel de significancia alfa $=0,05$ se observó que este último es mayor, llegando a la conclusión de rechazo de la hipótesis nula, y se concluyó que las actividades de la gestión formativa se relaciona significativamente con la satisfacción de 
necesidades de la Escuela de Posgrado de la Universidad Nacional José Faustino Sánchez Carrión, Huacho-2017.

Tabla 3. Prueba Chi cuadrado de la Gestión Administrativa

\begin{tabular}{lc}
\hline \multicolumn{1}{c}{ Estadísticos } & Valor \\
\hline Chi_Cuadrado & 16,35 \\
Sig (bilateral) & 0 \\
Nivel de significancia & 0,05 \\
Coeficiente de contingencia & 0,59 \\
Coeficiente de correlación & 0,77 \\
Número de evaluados & 264 \\
\hline
\end{tabular}

En la tabla 3 se observa la correlación entre la dimensión gestión administrativa y satisfacción de necesidades el cual fue 0,77 lo que indicó una alta correlación positiva y significativa, así mismo al comparar la sig.(bilateral) $\mathrm{p}$ valor $=0$ con el valor del nivel de significancia alfa $=0,05$ se observó que este último es mayor, llegando a la conclusión de rechazo de la hipótesis nula, y se concluyó que las actividades de la gestión administrativa se relaciona significativamente con la satisfacción de necesidades de la Escuela de Posgrado de la Universidad Nacional José Faustino Sánchez Carrión, Huacho -2017.

Tabla 4. Prueba Chi cuadrado de la Gestión de la Investigación.

\begin{tabular}{lc}
\hline \multicolumn{1}{c}{ Estadísticos } & Valor \\
\hline Chi_Cuadrado & 16,12 \\
Sig (bilateral) & 0 \\
Nivel de significancia & 0,5 \\
Coeficiente de contingencia & 0,55 \\
Coeficiente de correlación & 0,72 \\
Número de evaluados & 264 \\
\hline
\end{tabular}

En la tabla 4 se observa la correlación entre la dimensión gestión de investigación y satisfacción de necesidades el cual fue 0,72 lo que indicó una alta correlación positiva y significativa, así mismo al comparar la sig.(bilateral) $\mathrm{p}$ valor $=0$ con el valor del nivel de significancia alfa $=0,05$ se observó que este último es mayor, llegando a la conclusión de rechazo de la hipótesis nula, y se concluyó que Las actividades en la gestión de investigación se relacionan significativamente con la satisfacción de necesidades de la Escuela de Posgrado de la Universidad Nacional José
Faustino Sánchez Carrión, Huacho - 2017.

\section{DISCUSIÓN.}

En la Política Nacional de Modernización de la Gestión Pública al 2012:

\begin{abstract}
La razón de ser de la gestión pública es servir a los ciudadanos. Ello significa que el Estado y sus entidades deben definir sus prioridades $e$ intervenciones a partir de las necesidades ciudadanas y en función de ello, establecer las funciones y los procesos de gestión que permitan responder más y mejor a esas necesidades con los recursos y capacidades disponibles en cada momento presente (PCM, 2012).
\end{abstract}

Como consecuencia de los cambios en la gestión pública, las entidades de educación superior como parte de la modernización del estado afrontan una reforma de estructura y funcionamiento situándolas en un espacio expectante al dar un viraje en la percepción de la atención de calidad al estudiante, ello queda demostrado en la nueva Ley Universitaria $\mathrm{N}^{\circ} 30220$, generando en la atención el ordenamiento de las actividades orientadas a la organización por procesos donde los inputs y el outputs se convierten en el soporte de los cambios.

Por otra parte, el aprendizaje en la gestión por procesos de las entidades de educación superior Universitaria según Pérez, (2012). Señala que el "dilema" es el primer obstáculo con el que se encuentra una organización que desee adoptar este enfoque. Ante este "dilema", es necesario recordar que los procesos ya existen dentro de una organización, de manera que el esfuerzo se debería centrar en identificarlos y gestionarlos de manera apropiada. Al respecto la presente investigación presenta como resultado la siguiente identificación de procesos misionales: gestión formativa, gestión administrativa y gestión de la investigación, orientados al mejoramiento de la calidad educativa a nivel estudios de posgrado.

El enfoque que como profesional docente se le dé al proceso de enseñanza tendrá un peso significativo y repercutirá de igual manera en el proceso de aprendizaje de los alumnos. Pensar en la manera en que se llevará a cabo el acto educativo es pensar en la importancia que tiene, especialmente hoy en día, la educación. Si educar ya no implica transferir conocimiento, si educar ya no implica "dar clase", si educar ya no significa que un grupo heterogéneo de alumnos escuche atentamente a su docente, entonces supone 
proponer herramientas y procesos para grupo de educandos heterogéneos, diversos con capacidades talentos y proclividades múltiples.

En relación a las actividades de la gestión formativa y la satisfacción de necesidades de laEscuela de Posgrado de la Universidad Nacional José Faustino Sánchez Carrión

\begin{abstract}
El segundo Objetivo Estratégico Sectorial (OES 02)del Plan Estratégico Institucional de la Universidad Nacional José Faustino Sánchez Carrión menciona lo siguiente: Garantizar una oferta de educación superior técnica y universitaria que cumpla con condiciones básicas de calidad"; que busca garantizar a todos los jóvenes del país la oportunidad de acceder a un servicio educativo de calidad, que formen profesionales y técnicos de manera integral, y que permita la producción de conocimiento, ligado a la innovación, ciencia y tecnología que contribuya a la solución de los problemas nacionales y a los desafios del desarrollo sostenible"(PEI, 2017, p.11)
\end{abstract}

Una aproximación a la modernidad de la gestión por procesos sin duda es la construcción de un horizonte estratégico que alineé la gestión formativa con la satisfacción de los estudiantes de posgrado al recibir el servicio educativo, consecuentemente las actividades de la gestión formativa se relacionan significativamente con la satisfacción de necesidades de la Escuela de Posgrado de la Universidad Nacional José Faustino Sánchez Carrión.

Con relación las actividades de la gestión administrativa y su relación con la satisfacción de necesidades de la Escuela de Posgrado de la Universidad Nacional José Faustino Sánchez Carrión Encontramos en PEI de la institución que el OEI 04: Mejorar la gestión institucional en la Universidad Nacional José Faustino Sánchez Carrión:

La universidad busca mejorar los procesos de planificación, conducción, supervisión, evaluación y de coordinación dentro de la gestión administrativa central, las unidades académicas, con el soporte de la implementación de las estrategias en tecnología e información, así como del fortalecimiento de capacidades. (p.9)

Es evidente que la entidad necesita constantemente adaptar y mejorar sus procesos, pese a que frecuentemente están frenadas por aplicaciones y sistemas que no están preparados para explotar nuevas oportunidades y adaptarse a los cambios de forma ágil. Al respecto los resultados nos muestran que la universidad al declarar la mejora en la gestión demuestra actitud de cambio; y consecuentemente implantar nuevas estrategias permite correlacionar en forma significativa las necesidades para lograr la satisfacción como parte de la gestión de calidad en los servicios que brinda la Escuela de Posgrado de la Universidad Nacional José Faustino Sánchez Carrión. En relación a las actividades en la gestión de investigación y su relación con la satisfacción de necesidades de la Escuela de Posgrado de la Universidad Nacional José Faustino Sánchez Carrión, se observa a la práctica en investigación científica como el motor del desarrollo de un país como señala el CONCYTEC (2014), en el documento Situación de la formación de capital humano e investigación en las universidades peruanas. II Censo Nacional Universitario 2010:

\begin{abstract}
... un país que busque mantener un nivel de PBI per cápita similar al determinado como meta para el bicentenario del Perú (2021), debería mantener en promedio una cuantía de 1,600 investigadores por millón de habitantes. Sobre la base de dicho supuesto, se ha estimado que para el año 2021 el Perú requerirá aproximadamente 17, 500 investigadores con doctorado en las áreas de ciencias básicas e ingeniería. Tomando en cuenta la cifra actual de investigadores registrados en el CONCYTEC, la brecha al 2021 será de alrededor de 15,700 investigadores con grado de doctor...". (p.6)
\end{abstract}

Consecuentemente es evidente, que en los últimos tiempos la investigación científica y calidad traducida en satisfacción de las necesidades del posgrado los resultados del estudio corroboran que se relacionan significativamente como se muestra en la alta correlación 0,72 y significancia alfa $=0,05$ en las variables analizadas.

\section{CONCLUSIÓN}

Las actividades de la gestión formativa se relacionan significativamente con la satisfacción de necesidades de la Escuela de Posgrado de la Universidad Nacional José Faustino Sánchez Carrión.

Las actividades de la gestión administrativa se relacionan significativamente con la satisfacción de necesidades de la Escuela de Posgrado de la Universidad Nacional José Faustino Sánchez Carrión.

Las actividades en la gestión de investigación se relacionan significativamente con la satisfacción de necesidades de la Escuela de Posgrado de la Universidad Nacional José Faustino Sánchez Carrión. Finalmente se concluyó que la identificación de procesos misionales se relaciona 
significativamente con la gestión de calidad en la Escuela de Posgrado de la Universidad Nacional José Faustino Sánchez Carrión, Huacho - 2017.

\section{REFERENCIA BIBLIOGRÁFICA}

Briones, G. (2002). Metodologa de la Investigación Cuantitativa en las Ciencias Sociales. (A. editores, Ed.) Bogotá, Colombia.

CONCYTEC (2014): Situación de la formación de capital humano e investigación en las universidades peruanas. II Censo Nacional Universitario 2010. file://C:/Users/USER/Downloads/Diagnosticodeun iversidades_versionfinal.pdf

Duque, A. V. (2013). Metodologia de Intervención Social. Manizales, Colombia: Epi-Logos.

Goleman, D. (2010). El lider resonante crea más (Tercera ed.). Barcelona, España: Debolsillo.

Hernandez, S, Fernandez C.R. \& Baptista L.P.(2006) Metodología de la investigación. Editorial Mc. Graw Hill. 4ta Edición.

PCM, S. d. (2012). Política de Modernización de la Gestión del Estado al 2021. Consejo de Ministros, Lima.

Pérez, J. (2012). Gestión por Procesos (quinta ed.). Madrid, España: ESIC Editorial.
SUNEDU. (2014). Ley $\mathrm{N}^{\circ} 30220$ - Ley Universitaria. SUNEDU. Lima, Perú: Superintendencia Nacional de Educación Superior Universitaria. Recuperado el 08 de Noviembre de $2017, \mathrm{de}$ https://www.sunedu.gob.pe/nueva-ley-universitaria30220-2014

Universidad Nacional José Faustino Sánchez Carrión (2017): Plan Estratégico Institucional (2017-2019). $\mathrm{O}$ b t e $\mathrm{n} \mathrm{i} \mathrm{d} \mathrm{o}$ d e http://www.unjfsc.edu.pe/archivos/emergente/PEI $\% 202017$ pdf 